\title{
Estrategias didáctico-pedagógicas para el abordaje socio-ecológico en ingeniería civil
}

Astelarra, Sofía* y Bochaton, Guillermo Iván*

\section{Resumen}

El objetivo del presente trabajo será describir y analizar la aplicación de una estrategia didáctica-pedagógica que propicie el abordaje socio-ecológico para el diseño y la planificación de un proyecto en ingeniería civil. Específicamente, caracterizamos la estrategia didáctica-pedagógica de «Simulación de un Estudio de Impacto Ambiental» (ESIA) en el marco curricular de la asignatura «Gestión Ambiental y Desarrollo Sustentable» (GAyDS) de la carrera de Ingeniería Civil de la Facultad Regional General Pacheco de la Universidad Tecnológica Nacional, Argentina. A modo de estructurar el artículo, primero definimos las perspectivas teóricas de análisis; segundo, el abordaje de la importancia de la Educación Ambiental dentro de la ingeniería civil; tercero, el contexto particular de la carrera en la que se sitúa esta propuesta. Seguidamente, describimos la estrategia didáctica-pedagógica, así como las reflexiones que suscita. Finalmente, sintetizamos la relevancia de la implementación de esta estrategia para el abordaje socio-ecológico de los proyectos de ingeniería civil.

Palabras clave: Educación Ambiental; estrategias didácticas-pedagógicas; abordaje socio-ecológico; estudio de impacto ambiental; ingeniería civil

El presente artículo se desprende de las tareas docentes y de investigación de quienes escriben en la carrera de Ingeniería Civil de la Universidad Tecnológica Nacional, Facultad Regional General Pacheco (UTN-FRGP) y en el Proyecto de Investigación «Estudios y Formulación de Estrategias de Gestión Académica para la Formación de Ingenieros Civiles para el Desarrollo Sustentable en la Universidad Tecnológica Nacional», dirigido por la Mg. Lic. Liliana Ferranti y codirigido por el Ing. Osvaldo Russo. Recibido el 11/06/2020 y aceptado el 29/09/2020. Publicado el 02/05/2021.

DOI: https://10.33255/3262/793

Autoría: `Universidad Tecnológica Nacional, Regional General Pacheco (Argentina).

Contacto: sofiastelarra@gmail.com 
Astelarra, S.y Bochaton, G. I Estrategias didáctico-pedagógicas para el abordaje socio-ecológico en ingeniería civil

\title{
Didactic-pedagogical strategies for the socio-ecological approach in civil engineering
}

\begin{abstract}
The objective of this work will be to describe and analyse the application of a didacticpedagogical strategy that favours the socio-ecological approach for the design and planning of a project in civil engineering. Specifically, we characterize the didacticpedagogical strategy of «Simulation of an Environmental Impact Study» (EsIA) in the curricular framework of the subject «Environmental Management and Sustainable Development» (GAyDS) of the Civil Engineering career of the General Pacheco Regional School. from the National Technological University, Argentina. In order to structure the article, we first define the theoretical perspectives of analysis, second the approach to the importance of environmental education within civil engineering, third the particular context of the career in which this proposal is situated. Next, we describe the didacticpedagogical strategy, as well as the reflections it elicits. Finally, we synthesize the relevance of the implementation of this strategy for the socio-ecological approach of civil engineering projects.
\end{abstract}

Keywords: Environmental Education; didactic-pedagogical strategies; socio-ecological approach; Environment Effect investigation; civil Engineering

\section{Estratégias didático-pedagógicas para a abordagem socioecológica na engenharia civil}

\section{Resumo}

O objetivo deste trabalho será descrever e analisar a aplicação de uma estratégia didático-pedagógica que propicie uma abordagem socioecológica para a concepção e planejamento de um projeto de engenharia civil. Especificamente, caracterizamos a estratégia didático-pedagógica de «Simulação de um Estudo de Impacto Ambiental» (EsIA) no âmbito curricular da disciplina «Gestão Ambiental e Desenvolvimento Sustentável» (GAyDS) do curso de Engenharia Civil da Faculdade Regional General Pacheco da Universidade Tecnológica Nacional, Argentina. Para estruturar o artigo, definimos primeiro as perspectivas teóricas de análise, em segundo lugar a abordagem da importância da educação ambiental dentro da engenharia civil, terceiro o contexto particular da carreira em que esta proposta se situa. Em seguida, descrevemos a estratégia didático-pedagógica, bem como as reflexões que ela suscita. Por fim, sintetizamos a relevância da implementação desta estratégia para a abordagem socioecológica dos projetos de engenharia civil.

Palavras-chave: Educação Ambiental; estratégias didático-pedagógicas; abordagem socioecológica; Estudo de impacto ambiental; engenharia civil 


\section{Introducción}

El objetivo del presente trabajo será describir y analizar la aplicación de una estrategia didáctica-pedagógica que propicie el abordaje socio-ecológico para el diseño y la planificación de un proyecto en ingeniería civil. Específicamente, caracterizamos la implementación de la estrategia didáctica-pedagógica de «Simulación de un Estudio de Impacto Ambiental» (EsIA) en el marco curricular de la asignatura electiva «Gestión Ambiental y Desarrollo Sustentable» (GAyDS) de la carrera de Ingeniería Civil de la Facultad Regional General Pacheco de la Universidad Tecnológica Nacional, Argentina.

Entendemos la estrategia didáctica como aquella que comprende los métodos, las técnicas y los procedimientos favorecedores del proceso de enseñanza-aprendizaje, es decir, todos aquellos enfoques y modos de actuar dentro del espacio áulico (Carrasco, 2004; Ferranti, 2019).

La propuesta metodológica utilizada para este trabajo es descriptiva y analítica, basada en la sistematización de la práctica docente, la descripción de la estrategia didáctica-pedagógica, el contexto en el cual se aplica y las reflexiones teórico-pedagógicas al respecto.

A modo de estructurar el artículo, primero definimos las perspectivas teóricas de análisis; segundo, el abordaje de la importancia de la Educación Ambiental dentro de la ingeniería civil; tercero, el contexto particular de la carrera en la que se sitúa esta propuesta. Seguidamente, describimos la estrategia didáctica-pedagógica, así como las reflexiones que suscita. Finalmente, sintetizamos la relevancia de la implementación de esta estrategia para el abordaje socio-ecológico de los proyectos de ingeniería civil en las conclusiones.

\section{Perspectivas teóricas}

A partir de mediados del siglo XX, las cuestiones ambientales y la noción de crisis ambiental o ecológica se han ido instalando en los discursos, las percepciones y la sensibilidad social, cobrando primordial relevancia a nivel global-local tanto en las áreas de investigación científica como en las agendas y políticas gubernamentales, a la vez que progresivamente se ha incorporado en carreras o profesiones como la ingeniería civil.

Según Leff y Porto Gonçalves (2014; 2004), la crisis ambiental anuncia el límite de una modalidad de relación de la humanidad con la naturaleza que es destructiva de las condiciones de existencia que posibilitan la vida misma en el planeta. Como advierten: 
La crisis ambiental es una crisis civilizatoria, una crisis de los modos de comprensión, de cognición y de producción de conocimientos que a través de la hegemonía dominante ha construido un mundo insustentable. La crisis ambiental sorprendió a la humanidad encaminada hacia el progreso como ideal del lluminismo de la Razón y de la racionalidad científica-tecnológica-económica de la modernidad, inscrita en la era de la imagen del mundo (Leff, 2014: 16).

Estos modos de comprensión, de cognición y de producción -en sentido amplio- están imbricados en las diferentes racionalidades, apropiación y significación social de la naturaleza, imaginarios sociales, etc., que portan tanto los sujetos como los actores sociales. Esta crisis ambiental nos interpela a transformar los modos de conocimiento, las prácticas del conocer y comprender que están imbricadas en las maneras de intervenir en la realidad.

En este sentido, sostenemos lo planteado por Enrique Leff (1998) en cuanto a la necesidad de construir otra racionalidad social que integre la cuestión ambiental. Para realizarlo se torna fundamental la incorporación de la Educación Ambiental (EA) en todos los niveles de la educación. Para lo cual se promueve la EA que:

Se funda en dos principios básicos: 1) Una nueva ética que orienta los valores y los comportamientos hacia objetivos de sustentabilidad ecológica y justicia social. 2) Una nueva concepción del mundo como sistemas complejos, la reconstitución del conocimiento y el diálogo de saberes (Leff, 1998a: 9).

La Educación Ambiental debe estar orientada a cuestionar, reflexionar y producir conocimientos vinculados a superar la visión fragmentada de la realidad incorporando el pensamiento de la complejidad, desde el enfoque del campo de la Teoría de Sistemas propuesta por Morin y retomada por Leff, entre otros autores. Esta propone concebir lo estudiado o «la realidad» «como asociación combinatoria de elementos diferentes», en tanto partes integrantes de sistemas abiertos. Lo que implica que su «existencia y estructura dependen de una alimentación exterior (...), no solamente material-energética, sino también organizacional-informacional» (Morin, 1990: 41).

Esta noción de sistemas complejos abiertos al flujo de materia-energías externas e internas a la par que el flujo organizacional-informacional es clave. Implica abandonar la concepción tradicional y fragmentaria de los sistemas en tanto circuitos cerrados, por ejemplo, la economía por fuera de lo ambiental, lo social y lo cultural. En este sentido, es necesario comprender desde la teoría y la práctica 
la especificidad de los procesos socioambientales como sistemas complejos: por una parte, se trata de aprehender una realidad multidimensional en la que confluyen procesos no lineales, de diferentes niveles de espacialidad y temporalidad, con diferentes formas de interdependencia, de donde emergen nuevos procesos que establecen variadas sinergias y retroalimentaciones, tanto positivas como negativas (Leff, 1998b: 194).

Siguiendo a Swyngedouw (2009) entendemos que:

Los ambientes producidos son resultados históricos específicos de procesos socio-biofísicos. La mayoría de los procesos sociales y las condiciones socio-ecológicas (ciudades, sistemas de producción agrícola o industrial y similares) son invariablemente sostenidos y organizados a través de una combinación de procesos sociales por un lado (como las relaciones capital / trabajo y las formas de organización del trabajo) y procesos metabólicos-ecológicos (es decir, la transformación biológica, química o física de los recursos «naturales», generalmente organizados a través de una serie de tecnologías interconectadas) por el otro (Heynen et al., 2005, citado en Swyngedouw, 2009: 2).

La EA propicia la construcción de esta visión integral del ambiente considerándolo en sus múltiples y complejas combinatorias de dimensiones tanto físicas, culturales, bióticas, como tecnológicas, históricas, políticas, económicas, éticas, entre otras, así como entender que dicha combinatoria e interacción está configurada o atravesada por relaciones de poder a lo largo del tiempo, puestas en práctica por diferentes actores sociales. Por ello, consideramos primordial enfatizar que estos procesos son construcciones sociales e históricas que se ajustan a las necesidades biofísicas de cada época. Este cambio de modo de construcción de conocimiento es necesario para actuar de manera ética desde una racionalidad ambiental que promueva un futuro sustentable (Leff, 1998b; SAyDS, 2009).

\section{Importancia de la Educación Ambiental para el Desarrollo Sustentable en ingeniería}

Las/os profesionales de la ingeniería, y en particular de la ingeniería civil, en tanto proyectistas, constructores y operadores de grandes obras que transforman el territorio, constituyen actores centrales de la tensión existente entre desarrollo y ambiente.

Swyngedouw, retomando a Harvey, considera que: 
Los entornos hidráulicos son construcciones socio-físicas que se producen de manera activa e histórica, tanto en términos de contenido social como de cualidades físico-ambientales. Por lo tanto, no hay nada a priori antinatural en entornos construidos como presas, sistemas de riego, infraestructuras hidráulicas, etc. (Harvey, 1996, en Swyngedouw, 2009: 2).

Esta manera de comprender los entornos hidráulicos nos permite interrogar no solo a la ingeniería hidráulica en particular, sino a la ingeniería civil en general como disciplina transformadora del territorio.

Retomamos la noción de territorio de la geografía crítica que lo concibe como una construcción social e histórica, situada en un espacio concreto o espacio geográfico (Lefebvre, 1974; Harvey, 2000; Haesbaert, 2011). Cuando un tipo de relación social modela (controla) material y simbólicamente una porción de la superficie terrestre, decimos que se ha configurado un territorio, o sea, que tal relación social se ha territorializado, desterritorializando posiblemente otro tipo de relaciones. A su vez, un territorio específico es una producción contingente del espacio, y es resultado de conflictos, cooperaciones, superposiciones y exclusiones, de distintas relaciones sociales que buscan anclarse espacialmente generando coexistencias más o menos subordinadas. O bien, imposibilitando las coexistencias.

De modo que la ingeniería civil se propone realizar un tipo de apropiación material y simbólica sobre un territorio en el cual ya están situadas ciertas territorialidades. Motivo por el cual alertamos respecto a tener en cuenta que el tipo de intervención sobre los entornos hidráulicos u otros genera transformaciones y cambios.

En este sentido, entendemos que los procesos de cambio socioambiental no son ni social ni ecológicamente neutrales, en tanto las acciones «producen una serie de cambios sociales y ambientales tanto habilitadoras como discapacitantes» (Swyngedouw, 2009). Es decir, las mejoras que puedan producirse para determinados sectores pueden conducir a un empobrecimiento de condiciones sociales y ambientales en otros sectores.

Por otra parte, es menester considerar que la noción de desarrollo sostenible o sustentable es polisémica; según quién, cómo, dónde y cuándo se utilice será el paradigma en el cual se inscribe (Di Pace et al., 2012; Gudynas, 2004). Conocer los diferentes paradigmas ambientales en los cuales se enmarca la noción de Desarrollo Sustentable o Sostenible es necesario para comprender las tensiones entre el desarrollo y el ambiente y los tipos de sustentabilidad (Gudynas, 2004; Gallopin, 2003), a la par que las actuaciones profesionales y las consecuencias socio-ecológicas derivadas de estas. 
Esto es fundamental a la hora de proyectar obras de ingeniería, dado que estas incrementan la complejidad ambiental cuando se realizan; por lo tanto, comprender y analizar esos territorios se vuelve fundamental para promover una ingeniería civil para el desarrollo sustentable. El desconocimiento de conceptos ambientales básicos en el ejercicio profesional implica poner en riesgo los bienes comunes de la naturaleza y, en consecuencia, la vida humana-no humana. Por lo tanto, las universidades se encuentran con la responsabilidad de incorporar en sus contenidos y diseños curriculares este enfoque de la complejidad en la formación de los/as profesionales de la ingeniería civil.

Complementariamente, Ferranti fundamenta que:

Los contenidos curriculares constituyen sin duda la base indispensable, pero para tomar decisiones profesionales acordes a los principios del desarrollo sostenible, hacen falta otras competencias vinculadas a lo que podríamos denominar aprendizaje social. Esto supone enfocar los contenidos teniendo en cuenta el contexto, los aspectos internacionales, las prioridades locales y fundamentalmente, desarrollar la capacidad para tomar decisiones no sólo en base a criterios estrictamente técnicos sino considerando a la comunidad que se verá involucrada en estas decisiones (Ferranti y otros, 2019).

En definitiva, la EA para el DS en ingeniería civil requiere emplear propuestas pedagógicas y métodos participativos que permitan construir un pensamiento complejo, crítico, y reflexionar sobre situaciones reales, fomentando el abordaje interdisciplinario (Ferrer et al., 2008).

\section{Contexto curricular en la UTN-FRGP}

En el campo de la ingeniería civil, el concepto de sustentabilidad, desarrollo sustentable o sostenible comienza a ser incorporado recientemente, primero desde lo discursivo, para proyectarse en los procesos de formación académica. El «Congreso Mundial de Ingeniería 2010», realizado en la ciudad de Buenos Aires, tuvo como objetivo:

Impulsar el trabajo conjunto de los ingenieros con las fuerzas productivas para lograr la convergencia que fortalezca su cooperación conducente al desarrollo sostenible, con protección ambiental, equidad e inclusión social, tal como lo definió la Cumbre Mundial de las Naciones Unidas en 1992 en Río de Janeiroํㅗ. 
Desde Argentina, en concordancia con esta preocupación, el Consejo Federal de Decanos de Ingeniería realizó un aporte al Congreso y destacó la necesidad de formar profesionales con una visión sistémica para el Desarrollo Sustentable (CONFEDI, 2010). Desde entonces, se ha incrementado la incorporación en el currículum académico de asignaturas tendientes a ello, así como la promoción de prácticas preprofesionales que lo contemplen.

La carrera de Ingeniería Civil de la UTN-FRGP fue pionera en incorporar en 2008 la asignatura Gestión Ambiental y Desarrollo Sustentable (GAyDS)ํ. Desde entonces, se enmarcan los contenidos teórico-prácticos dentro del perfil profesional de la carrera de Ingeniería Civil de la Facultad Regional General Pacheco de la UTN, en el cual se sostiene que cada estudiante:

deberá reconocer las cuestiones políticas, económicas, institucionales y sociales como elementos centrales del complejo sistema en que habrá de desempeñarse profesionalmente. Desde su formación ética bregará por sostener los principios del desarrollo sustentable propiciando la transformación productiva en un marco de respeto por el equilibrio ecológico y por la distribución equitativa de los recursos involucrados (Perfil del Ingeniero/a Civil de la UTN FRGP, Resolución Departamental n. ${ }^{\circ}$ 01/07).

Partiendo de este perfil singular de esta facultad, consideramos que dichos principios permiten comprender la cuestión ambiental y el desarrollo sustentable en los contextos de transformación social actual, enfatizando en el rol del ingeniero/a civil respecto a estos, así como la importancia ético-profesional de incorporar contenidos que posibiliten el trabajo interdisciplinario, la acción reflexiva y flexible de las/os ingenieras/os civiles que garanticen una inserción, adaptación y participación en contextos actuales.

GAyDS es asignatura electiva del último año de la carrera, que se desarrolla en seis horas cátedra semanales durante el primer cuatrimestre del calendario académico. En paralelo se dicta la asignatura «Proyecto Final» de la carrera. Este es el resultado de la interacción entre el sector académico, estudiantes y docentes, con organismos gubernamentales, tales como municipios, o bien organizaciones sociales o de la sociedad civil con las que las/os estudiantes estén familiarizados. De manera que el Proyecto Final surge de una necesidad o inquietud de sectores gubernamentales, organizaciones sociales o, en menos casos, propuestas que las/os estudiantes realizan a estos actores sociales. Dichos proyectos son ejercicios didáctico-pedagógicos, parte del proceso para terminar la carrera estudiantil y primera aproximación profesional. 
En ese contexto, de la integración de las asignaturas GAyDS y Proyecto Final resulta la confección de la «Simulación de EsIA» de dicho proyecto. Por lo tanto, este ejercicio académico es de notable relevancia para construir una mirada socioecológica que incida en el ejercicio profesional de la ingeniería civil.

\section{Perspectiva didáctico-pedagógica}

La perspectiva didáctico-pedagógica adoptada por la cátedra considera el aprendizaje como una construcción social e históricamente situada, a la vez que aborda el proceso de enseñanza y aprendizaje desde una perspectiva sociopolítica (Malagón Patiño, 2018). Los objetivos principales de la asignatura son que las/os estudiantes adquieran, profundicen sus conocimientos y capacidades respecto a las cuestiones ambientales en general, la gestión ambiental y el desarrollo sustentable en particular. Para ello, la apuesta teórico-metodológica de la cátedra es generar un espacio áulico en el cual sea posible interrogar la función ético-política de la ingeniería civil en función de un futuro sustentable.

El equipo de cátedra considera que el conocimiento es una práctica social que se construye en el hacer, la investigación, la formulación de preguntas problemáticas, y la observación crítica de la realidad en la que vivimos y actuamos profesionalmente.

Esta práctica social situada en el espacio áulico se genera a partir de la relación de enseñanza-aprendizaje entre docentes y estudiantes, centrada en el estudiante. Dicha situación de aprendizaje requiere un momento de «desestabilización» y otro de partida denominado «punto de apoyo». Esto significa que para aprender partimos de un lugar de referencia sobre el cual se trabaja, el punto de apoyo, lo cual es nodal ya que implica trabajar con los saberes, experiencias, sentimientos, expectativas, deseos de los/as estudiantes. El segundo momento implica desestabilizar ese punto para aprender algo nuevo, introduciendo conceptos teóricos. Para ello, será necesario generar un espacio propicio para la circulación de la palabra, la participación de los/las estudiantes, de modo que podamos reflexionar-producir conjuntamente desestabilizando los saberes previos que todos/todas poseemos.

La tarea de la/los docentes de la cátedra, en las clases teóricas y prácticas será orientar el aprendizaje buscando generar una relación de enseñanza-aprendizaje en la que se construya conocimiento, se elaboren y se reelaboren preguntas y problemas, y se reflexione sobre las prácticas sociales en general y de los/las ingenieros/as civiles en particular. 
Torres Pernalete (2012) destaca que entre las propuestas metodológicas que se han reportado como exitosas y recomendadas en universidades del mundo entero, se encuentran: el aprendizaje colaborativo, el aprendizaje basado en problemas (ABP), el aprendizaje orientado a proyectos (POL), el aprendizaje basado en casos y otros con igual potencial (Pernalete citado en Ferranti, 2019).

En la búsqueda por generar estrategias o herramientas didácticas-pedagógicas que posibiliten el aprendizaje colaborativo mediante el ABP y el POL es que consideramos apropiadas la elaboración del EsIA y el empleo del método de la Matriz de Impacto Ambiental.

Dado que estamos en un contexto de formación profesional, proponemos la realización de una Simulación de EsIA como parte de la preparación para el futuro desempeño profesional. Entendemos esta estrategia didáctica como un tipo de estudio de caso; en tal sentido la consideramos como un instrumento educativo complejo (Wasserman, 1999). Esta nos resulta adecuada para la puesta en práctica de los dos objetivos de la EA ya explicitados. Por un lado, es un instrumento educativo que prepara a los/as estudiantes para comprender situaciones complejas; en otras palabras, comprender teórica y prácticamente el pensamiento de la complejidad. En segundo lugar, como mencionamos, esta Simulación se enmarca en la realización del Proyecto Final que las/os estudiantes realizan en conjunto con actores gubernamentales y sociales y con diferentes docentes universitarios/as. Por tanto, es un ejercicio académico que no reviste carácter legal, aunque queda a disposición de los diferentes actores consultados. De modo que esta estrategia implica realizar un proceso de aprendizaje en el cual se pueda promover una nueva ética que orienta los valores y los comportamientos hacia objetivos de sustentabilidad socio-ecológicas.

Finalmente, la cátedra explicita que formalmente e idealmente estos estudios deben ser realizados por un equipo transdisciplinario que cuente con la presencia de diferentes disciplinas científicas, así como también deben contemplar la participación social de la población pasible de ser afectada. Una vez realizada esta aclaración para las/os estudiantes y quienes nos leen, damos inicio al proceso pedagógico-didáctico en el cual nos embarcamos.

\section{Proceso de elaboración de la simulación de estudio de impacto ambiental}

\section{Contexto legal de aplicación de los Estudios de Impacto Ambiental en Argentina}

En 1992, Argentina, en el marco de la Conferencia de las Naciones Unidas sobre el Medio Ambiente y el Desarrollo, firma la «Declaración de Río», cuyo principio 17 contempla la Evaluación de Impacto Ambiental. Consecuentemente, en la reforma constitucional de 1994, la nación incorpora el derecho al ambiente 
sano, y transfiere dominio de los recursos naturales a sus provincias, lo que derivó en la generación de marcos normativos en cada jurisdicción que regulan la Evaluación de Impacto Ambiental (EIA).

En 2002, la Ley General del Ambiente N. ${ }^{\circ}$ 25.675/02 establece presupuestos mínimos para conseguir una gestión sustentable del ambiente e incorpora el procedimiento de EIA como instrumento de política y gestión ambiental. A la fecha, no hay una ley de presupuestos mínimos de Evaluación de Impacto Ambiental.

En este sentido, se define a la EIA como el proceso cuyo objetivo principal consiste en identificar, predecir, evaluar y mitigar los potenciales impactos que un proyecto de obra o actividad puede causar al ambiente, en el corto, mediano y largo plazo. Consiste en un procedimiento técnico-administrativo que permite la toma de decisión informada por parte de la autoridad competente sobre la viabilidad ambiental de un proyecto y su gestión ambiental, previo a la ejecución del proyecto.

Por su parte, el EsIA constituye el documento técnico de carácter interdisciplinario que está destinado a predecir, identificar, valorar y corregir las consecuencias o efectos ambientales que determinadas acciones pueden causar sobre la calidad de vida del ser humano y su entorno (Conesa Fernández-Vítora, 1993). Es presentado por el proponente del proyecto, sea público o privado, a la autoridad ambiental correspondiente, y contiene:

la identificación del proponente, la descripción de proyecto, el diagnóstico o línea de base ambiental, el marco legal de cumplimiento, el resultado del análisis de alternativas, la identificación y valoración de los potenciales impactos ambientales que el proyecto puede causar en todas sus etapas, así como las medidas de mitigación para abordarlos que se estructuran en el Plan de Gestión Ambiental. (SAyDS, 2018)

El EsIA forma parte de la EIA y constituye una de las herramientas al servicio de la decisión, junto con la Audiencia Pública, por parte de la autoridad competente en la determinación de la Declaración de Impacto Ambiental (DIA). Todos estos son requisitos administrativos obligatorios para todos los proyectos u obras de ingeniería, pero también urbanas, productivas, gubernamentales, etc. De manera que nos resulta fundamental que las/os estudiantes conozcan en profundidad no solo la herramienta, sino el contexto y su utilidad social en pos de la sustentabilidad de los territorios en los cuales se desempeñarán profesionalmente. 


\section{Secuencia de estrategias didácticas previas}

Tal como planteamos, si comprendemos que los ambientes son resultado de procesos biofísicos y sociales a lo largo del tiempo, es necesario entender cómo se generan esas relaciones y desde qué paradigmas.

Para ello, en las primeras clases de la materia proponemos abordar conceptualmente esos procesos con sus respectivos paradigmas, e ir analizando los cambios, rupturas, continuidades y efectos sociales, políticos, económicos y ambientales de cada uno. Vamos contextualizando históricamente el surgimiento de la problemática ambiental en tanto asunto socialmente relevante, en primer lugar para las comunidades locales, organizaciones sociales o poblaciones que son afectadas por impactos ambientales; en segundo lugar, para académicas/os, científicas/os y profesionales que profundizan sus investigaciones al respecto, y finalmente, para los sectores gubernamentales encargados de realizar políticas públicas, control y regulación sobre estas problemáticas. El énfasis está puesto en comprender estos procesos en su complejidad, conflictividad y transformación, cuestionando visiones simplistas, dicotómicas y lineales de la historia-realidad socio-biofísica.

A partir de ese recorrido abordamos contenidos específicos asociados a: definir el desarrollo y desarrollo sustentable/sostenible, y los diferentes tipos de sustentabilidad que han surgido en función de las problematizaciones y conflictos sociales que se han suscitado. Finalmente, introducimos el concepto de territorio para comprender la dimensión espacial de esta relación socio-biofísica en la historia. Este concepto permite situar estos procesos asociados a que toda intervención humana se produce en un espacio ya habitado por humanos y no humanos, y que tiene efectos potenciantes o inhabilitantes, o bien, negativos o positivos (Swingedow, 2009).

Desde el inicio propiciamos el trabajo grupal, contemplando en este un trabajo personal de elaboración-reflexión para dar lugar a lo colectivo y a lo personal en el proceso de enseñanza-aprendizaje. Fomentamos armar grupos por actividad y otro grupo, que dura toda la cursada de la materia, para la elaboración de la Simulación del EsIA asociado al Proyecto Final.

Las/os estudiantes cuentan con bibliografía específica de los temas a trabajar durante la cursada, así como también material audiovisual, los que serán fundamentales para el desarrollo de la misma y para que se familiaricen e incorporen nuevos conceptos, problemas, realidades, etc. Además, se realizan actividades prácticas y de investigación que son acompañadas-supervisadas por la cátedra, así como compartidas durante las clases al resto del grupo. Esto último nos resulta fundamental porque en el proceso de investigación surgen preguntas y búsquedas propias de los/as estudiantes que favorecen a 
la incorporación de nuevos conocimientos, así como su profundización. Nuestro rol como docentes es facilitar y orientar este proceso de trabajo grupal.

Una vez concluida esta tarea generada en equipos por las/los estudiantes, estos deberán generar los informes que contemplen las consignas de cada trabajo y exponerlos ante la cátedra y el resto del curso. La apuesta pedagógica-didáctica se centra en que, en el espacio áulico, los/las estudiantes puedan establecer los vínculos entre el momento teórico y el momento práctico. En este sentido, es fundamental generar un espacio de discusión y reflexión a partir de trabajar con sus saberes previos y así incentivar una mayor apropiación de la teoría.

A partir de esta caja de herramientas conceptuales, de ejercicios prácticos y de preguntas reflexivas, iniciamos el trabajo de elaboración de la Simulación Estudio de Impacto Ambiental utilizando el método de matriz ponderada de Conesa Fernández-Vítora (1993).

\section{Momentos claves y etapas en la confección de la Simulación de Estudio de Impacto Ambiental}

En primera instancia, tal como mencionamos, reconocemos que en la confección del EsIA existen dos procesos paralelos pero integrados que tienen lugar en la asignatura Proyecto Final y en GAyDS; por lo cual, desde luego, se procura mantener los mismos grupos de trabajo para una y otra materia.

A continuación, describimos los momentos y las etapas que organizamos para dar inicio al trabajo de Simulación de EsIA, para luego dar cuenta cómo eso se va desplegando en el proceso de enseñanza-aprendizaje, las situaciones de ruptura y reelaboración tanto de las/os estudiantes como propias del equipo docente.

1. Descripción del entorno.

2. Primera identificación de efectos. Definición de factores.

3. Ponderación de factores.

4. Valoración de Impactos Ambientales.

5. Relectura y análisis de la matriz. Modificaciones de proyecto, medidas de mitigación. 


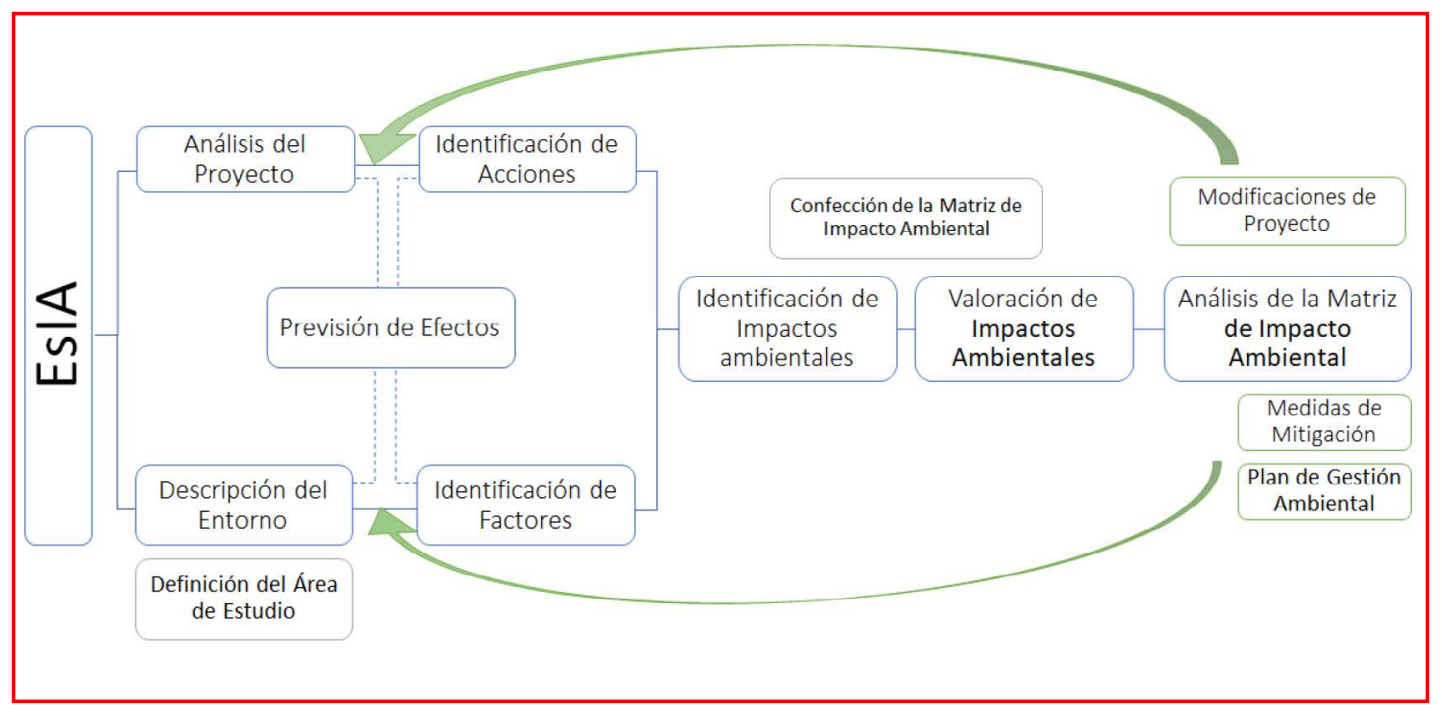

Figura 1. Etapas en la elaboración de un EsIA

Fuente: Elaboración propia en base a Conesa Fernández-Vítora, 1993.

\section{Descripción del entorno}

Por un lado, se da la elaboración y análisis del proyecto, desde su fundamentación, localización y características generales. Y por otro, la descripción del entorno y definición del Área de Estudio, que permitirá avanzar hacia un diagnóstico ambiental preliminar.

A partir de la localización del proyecto, las/os estudiantes realizan una búsqueda y jerarquización de la información necesaria para poder describir el entorno en el cual se insertará la obra, para empezar a caracterizar el ambiente tanto desde el medio físico-natural como del medio construido.

En esta primera etapa, nuestra tarea como equipo docente se centra en realizar una orientación bibliográfica y de fuentes de consulta en relación con los aspectos ambientales más relevantes del medio receptor, lo cual implica abordar de forma sintética aspectos del subsistema biótico e inerte del medio físico-natural, así como otros sociales, económicos, culturales y urbanos del medio construido, cuyos componentes interaccionan entre sí en un complejo sistema ambiental, atendiendo a un determinado recorte temporal.

Durante ese primer acercamiento al entorno, que permite comenzar a limitar el alcance del EsIA, se propone realizar un mapa de actores clave que estén vinculados o que podrían verse afectados por el proyecto, así como también se identifican organismos e instituciones que posean conocimiento sobre temas particulares en relación con algún aspecto relevante del medio receptor. Esta actividad facilita la caracterización del escenario social, identi- 
ficación de tipos de actores, perfil socioeconómico, grado de influencia, nivel de interés en el proyecto, etc.

Tras el análisis del proyecto y la caracterización preliminar del entorno, se trabaja en la definición inicial del Área de Estudio estableciendo sus límites geográficos. Lejos de establecer la delimitación simple mediante un círculo de radio amplio con centro en la localización del proyecto, planteamos que su determinación esté fundada en criterios físicos, sociales, económicos, demográficos, de coherencia hídrica o cualquier otro criterio adecuadamente justificado, comprendiendo que no es una tarea fácil y que cada especialista podría delimitar la unidad de estudio en función de su expertise.

Se propone, alcanzada esta instancia, una presentación informal de cada grupo en el que expongan los avances en la descripción del entorno, mapas de actores y área de estudio frente al curso con el objeto de abrir a consideración y debate lo realizado. En una dinámica de preguntas de parte del cuerpo docente y principalmente de las/os estudiantes, se verifica el intercambio de opiniones y de información que enriquecen la producción de cada trabajo.

De esta forma, se elabora el diagnóstico ambiental preliminar que sirve de base para la evaluación de los posibles impactos del proyecto.

\section{Primera identificación de efectos. Definición de factores}

Ya en la etapa previa, el conocimiento del medio receptor así como de las características del proyecto en sus distintas fases (construcción, operación, mantenimiento y cierre) va posibilitando una inicial interrelación prospectiva entre ambiente y proyecto. No obstante, consideramos adecuado sistematizar la previsión de efectos identificando factores ambientales pasibles de ser afectados e identificando acciones y actividades del proyecto que puedan afectarlos.

Listar las acciones del proyecto no presenta dificultad alguna para estudiantes de ingeniería civil cursando el último año de la carrera. En cambio, listar y definir los factores (o parámetros) que surgen de los distintos subsistemas ambientales descritos previamente conlleva la dificultad propia de la falta de formación en las diversas áreas disciplinares que se requiere que intervengan en la elaboración de los EsIA.

En consecuencia, se presta especial atención a la definición de los factores ambientales que varían de acuerdo con las características del entorno y a su relación con el proyecto, comenzando por la realización de un árbol cuyas ramas principales representan los subsistemas ambientales abriéndose en los respectivos componentes hasta ramificarse en sus parámetros de forma más desagregada. 


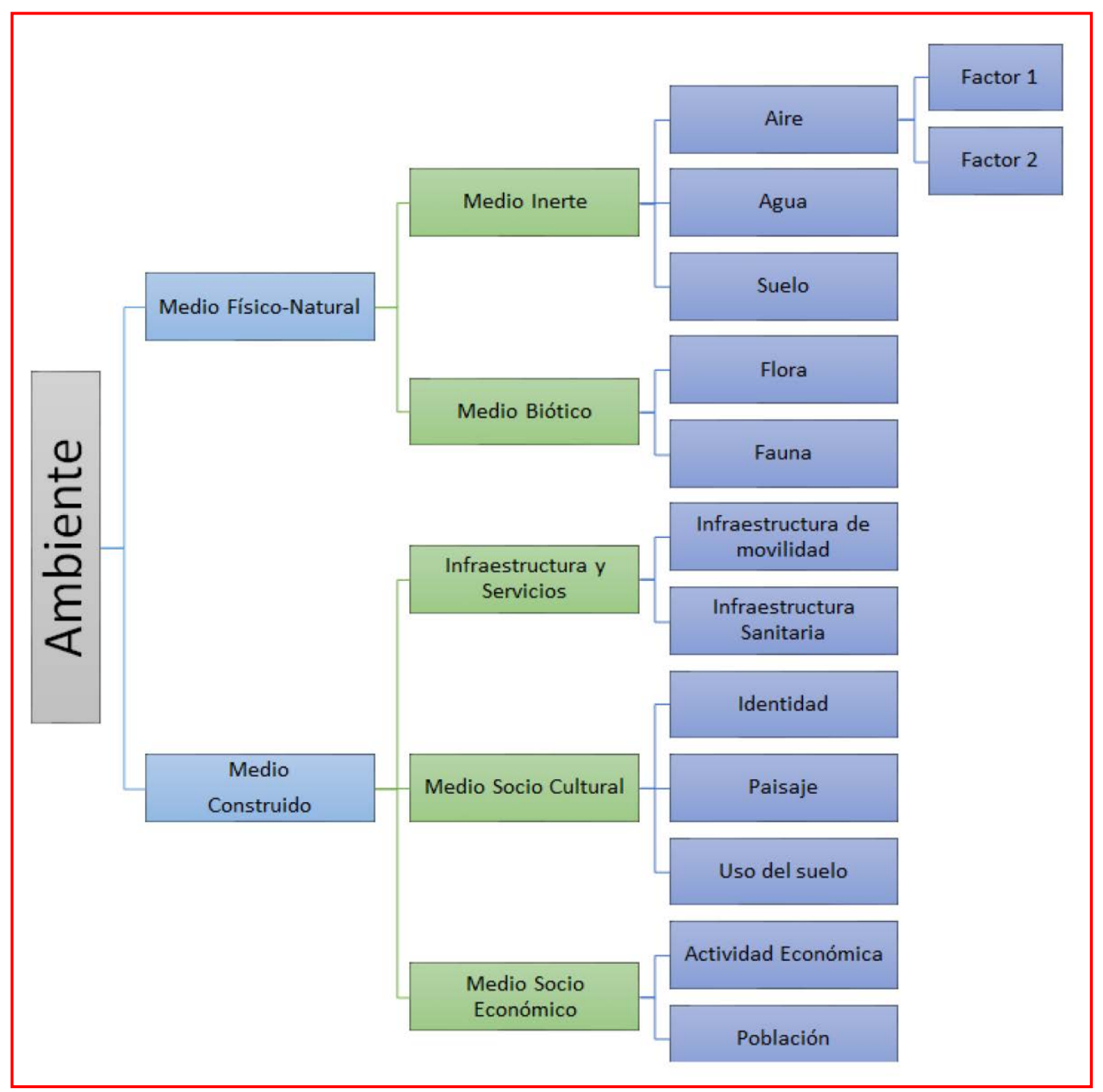

Figura 2. Árbol de factores ambientales

Fuente: Elaboración propia en base a Gómez Orea, 2002.

Al mismo tiempo que se enumeran los factores ambientales, se los define siguiendo criterios de representatividad y relevancia, considerando que sean excluyentes, de fácil identificación y, en la medida de lo posible, de fácil cuantificación. Aquí se trabaja de manera grupal, la tarea docente se centra en esclarecer dudas y apuntalar los conceptos.

Cerca del final de la clase, se expone el Árbol de Factores de cada proyecto para el intercambio con el resto de los grupos.

Al comenzar a relacionar la lista de Acciones con la lista de Factores, se obtiene una primera impresión de los efectos, tanto positivos como negativos, de unos sobre otros, permitiendo revisar en simultáneo los criterios que los 
definieron. Así puede resultar que algunos de los factores o acciones sean suprimidos, o bien, agregados.

Esta interrelación entre Acciones y Factores forma el esqueleto de la primera matriz, la Matriz de Identificación de Efectos; de esta forma, se consigue visualizar cuáles acciones tienen efectos sobre cuáles factores.

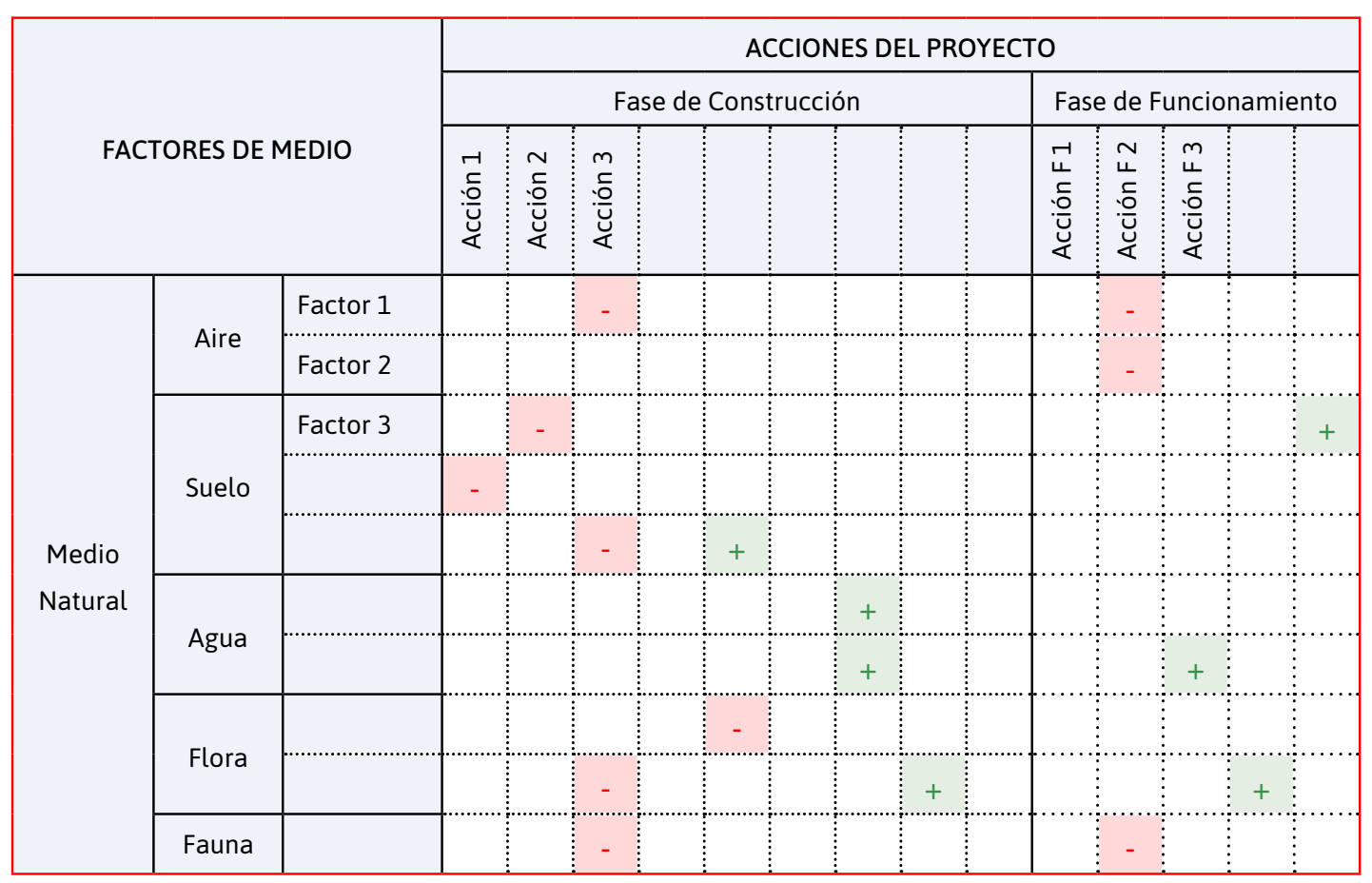

Figura 3. Matriz de Identificación de Efectos

Fuente: Fernández-Vítora, 1993.

\section{Ponderación de los factores}

En tanto los factores son elementos componentes del ambiente en el que se inscribe el proyecto, se vuelve necesario definir la participación de cada elemento dentro del sistema total. El peso de cada factor en el sistema se representa a través de Unidades de Importancia UIP, cuya suma total se establece en 1000.

La determinación de las UIP no es tarea sencilla, ya que demanda una argumentación que justifique cuáles factores pesan más que otros; por ello, se propone que cada grupo utilice el árbol de factores para debatir internamente y asignar UIP parciales por cada subsistema hasta llegar a cada factor. Aquí enfatizamos que la decisión debe estar justificada desde la perspectiva de la complejidad, simulando ser un equipo transdisciplinario, es decir, haciendo el ejercicio de pensar desde la biología, desde la sociología, entre 
otras perspectivas disciplinares. Inmediatamente, se comparan los factores y se ajustan en función de una nueva revisión.

Nuevamente, al final de la clase se elabora en el pizarrón una tabla que muestre de cada proyecto las UIP agregadas en componentes ambientales, o bien, en subsistemas, de forma tal que permita compararlas. De esta manera, es posible observar y revisar los criterios utilizados. Resulta esperable que el medio físico-natural tenga alta participación en proyectos que se emplazan en sitios poco antropizados; por el contrario, en proyectos que se enclavan en un tejido urbano existente, que la participación del medio construido sea altamente representativo.

Tras esta instancia, se realiza una primera presentación formal de los avances del EsIA. Se exige el uso de diapositivas digitales (PowerPoint o similar) y la regulación del tiempo de exposición, lo que favorece el proceso de aprendizaje personal y colectivo, dado que se expresan y argumentan los criterios que cada grupo ha realizado para la ponderación. Esto permite visibilizar la necesidad de analizar en profundidad cada proyecto de acuerdo con el entorno en el que se realizará, lo cual significa un primer momento de puesta en práctica de la EA, con relación al pensamiento complejo.

\section{Valoración de los impactos ambientales}

El siguiente momento clave transcurre durante la confección de la Matriz de Impacto Ambiental, particularmente en la valoración de los mismos utilizando el algoritmo planteado por el método de Conesa Fernández-Vitora (1993).

El algoritmo determina la Importancia del Impacto que cada Acción ejerce sobre cada Factor mediante la siguiente expresión: Imp $= \pm[3 I+2 E x+M o+$ $\mathrm{Pe}+\mathrm{Rv}+\mathrm{Si}+\mathrm{Ac}+\mathrm{Ef}+\mathrm{Pr}+\mathrm{Mc}$ ] que involucra dimensionar la Intensidad, la Extensión, el Momento, la Permanencia, la Reversibilidad, la Sinergia, la Acumulación, el tipo de Efecto, la Periodicidad y la Recuperabilidad del impacto de acuerdo con una escala cualitativa por cada elemento del algoritmo.

La Importancia del Impacto se construye mediante matrices auxiliares que organizan el abordaje separado de cada impacto, contemplando el nivel de intensidad y las características de cada efecto. El valor resultante permite caracterizarlos, siguiendo el método, en impactos irrelevantes, moderados, severos y críticos, y así obtener una primera valoración de los mismos. 


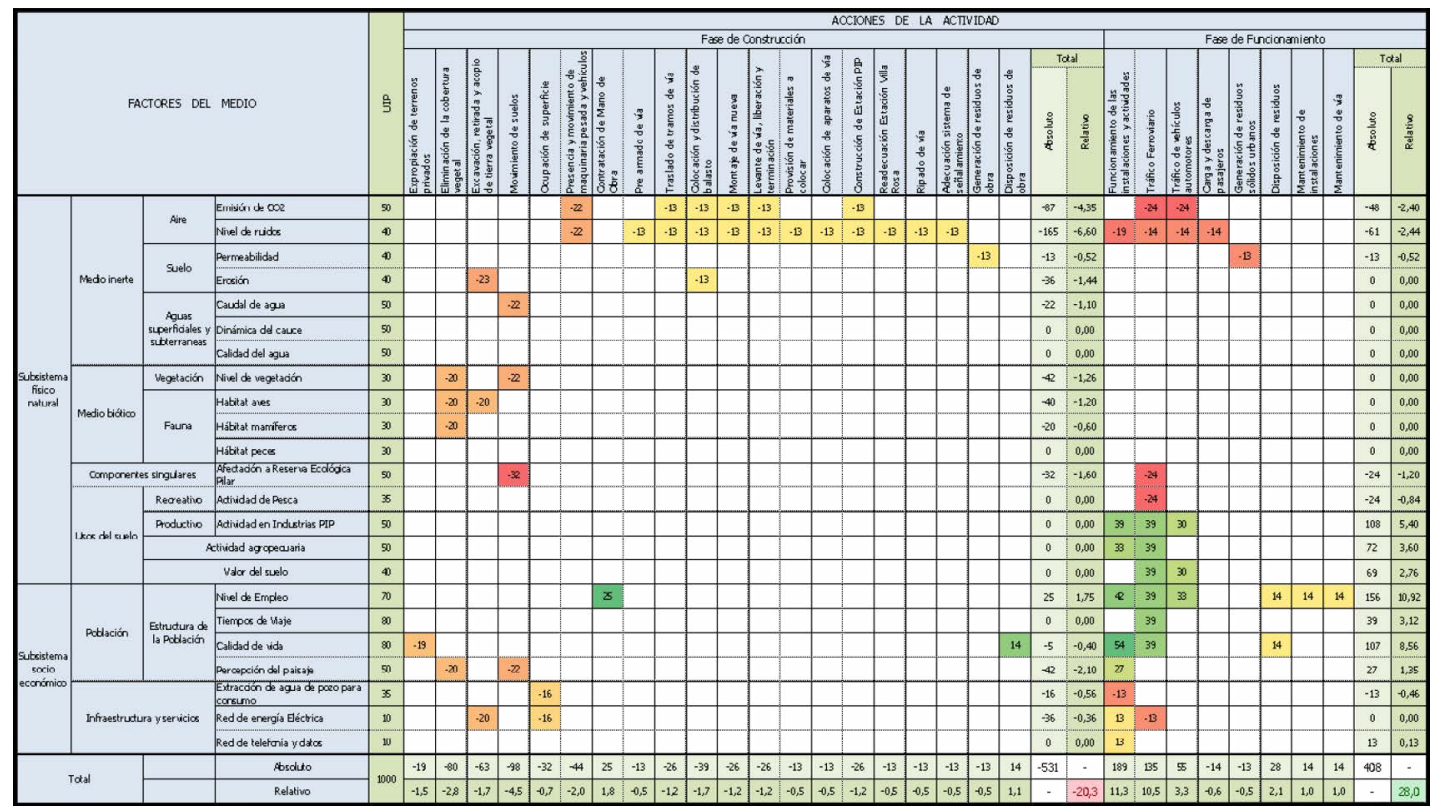

Figura 4. Matriz de Impacto Ambiental

Fuente: Elaboración propia.

\section{Relectura y análisis de la matriz. Modificaciones de proyecto, medidas de mitigación}

La lectura de la Matriz de Impacto Ambiental puede abordarse de diferentes modos; en consecuencia, permite establecer diversas relaciones entre sus componentes para interpretar la complejidad de los impactos ambientales.

Con el propósito de organizar la información que brinda, proponemos las siguientes lecturas: interrelación acción-factor de forma separada, acciones más impactantes en términos absolutos y relativos, factores más impactados, y el balance de impactos por cada fase de proyecto.

A continuación, les proponemos que realicen un apartado de «Conclusiones y lectura de la Matriz de Impacto», en el cual establezcan conexiones entre los valores principales arrojados por la matriz, considerando el proyecto en general, la acción y etapa del mismo. Finalmente, esperamos que las/os estudiantes realicen un abordaje de la Matriz en términos de una herramienta que posibilita interrogar respecto a la relación de una obra de ingeniería en un entorno socioambiental y en función de un desarrollo sustentable de la región. Este momento suele ser dificultoso dado que implica aprender a leer esos datos numéricos en función de la complejidad. Sin embargo, en su mayoría lo resuelven satisfactoriamente dado el trabajo procesual y acompañado.

Al atender a la complejidad ambiental de manera desagregada, pero a su vez, desde un enfoque sistémico, el análisis de la Matriz de Impacto Ambien- 
tal permite a las/os estudiantes repensar el proyecto. En primera instancia, surgen de los grupos distintas propuestas que pueden encuadrarse como implementación de medidas de mitigación.

Pero cabe destacar que, en varios casos, el ejercicio de repensar el proyecto en función de los impactos ambientales ha desembocado en modificaciones cruciales, reflejado en contemplar el uso de otras técnicas constructivas, otros materiales, hasta en planteo de alternativas. La mirada crítica hasta alcanzó en algún caso a cuestionar la necesidad y justificación de un proyecto.

Este es un proceso interesante que, si bien encuentra su final con el análisis de la matriz, sucede a medida que las/os estudiantes avanzan con la descripción del entorno y piensan en los posibles efectos que podría causar la ejecución del proyecto y su inserción en un ambiente determinado. En definitiva, desde la cátedra buscamos que en el futuro desempeño profesional la mirada crítica en términos socioambientales esté presente desde el momento en que surge la idea de un proyecto, atendiendo a su relación con otros proyectos, programas y planes territoriales.

Finalmente, el EsIA se entrega y expone en una presentación final frente a curso. El EsIA se evalúa, y si fuera necesario, se realizan las correcciones para su aprobación.

\section{Momentos de desestabilización de saberes, rupturas y reelaboración}

A partir de nuestros registros y de los comentarios de las/os estudiantes consideramos que hay varios momentos que pueden entenderse como «momentos de ruptura», es decir, aquellos en los cuales se desestabilizan los saberes previos con los nuevos conocimientos, abriendo nuevas preguntas, reflexiones y miradas crítico-reflexivas desde la complejidad ambiental.

La primera situación de ruptura sucede en el momento de «Descripción del entorno»; esta nos resulta la principal en el proceso de aprendizaje. En general, una primera respuesta de los/as estudiantes suele ser el traer una descripción escueta y superficial del entorno relevando aspectos vinculados al objetivo del proyecto. O bien, descripciones muy generales del municipio en el cual se localizará el mismo. En otras ocasiones, se confunde la descripción del entorno con el mapa de localización (incluso nos han traído un mapa catastral para dar cuenta del entorno del proyecto), a la vez que se confunde el objetivo del proyecto con su entorno. Estos problemas, lejos de ser errores en el aprendizaje, dan cuenta de una mirada fuertemente técnica y práctica que las/os estudiantes han incorporado a lo largo de la formación académica. Mirada necesaria para crear un proyecto y una obra de ingeniería, pero limitada para comprenderla y elaborarla desde la complejidad de la realidad actual. 
En este momento se produce una desestabilización de saberes previos. Las/os estudiantes vienen con un cúmulo de saberes técnicos y de nociones respecto a lo que les representa «lo social» y «lo ambiental», que está marcado por prejuicios sociales y paradigmas tecnicistas o economicistas que han naturalizado. Por lo tanto, este ejercicio práctico de descripción permite que consideren dimensiones y realidades que les eran invisibles. $\mathrm{O}$ bien, las consideran, pero no logran organizar una descripción, en dimensiones sociales, económicas, políticas y ambientales. Esta dificultad algunas veces termina por desanimarlos por lo que elaboran una descripción somera o desordenada para «cumplir con la consigna».

Para facilitar este proceso, elaboramos preguntas sugerentes. Por ejemplo, si la obra a proyectar es de reorganización del tránsito para revalorizar zonas urbanas o mejorar la circulación vial, las preguntas que realizamos son: «¿Cómo es el área donde se localiza el problema del tránsito que se quiere solucionar? ¿Es un área urbana, qué características tiene la zona aledaña? ¿Quiénes viven allí o qué sectores sociales se localizan? ¿Quiénes transitan por el área? ¿Qué actividades realizan? ¿Qué otras problemáticas pueden estar presentes? ¿Hay arbolados, flora o fauna en la zona? Describa en profundidad» (fragmento de Consigna TP3).

En concordancia con el ejemplo, no se trata solo de ver los árboles que están en la zona de trazado de las vías, sino de comprender que esos árboles son parte o han conformado en los años un corredor biológico urbano, en el que circulan aves e insectos que no son visibles fácilmente pero que tienen una función ecosistémica en el área urbana, además de ser parte del paisaje urbano que hace más agradable la vida social. De modo que si hay que desmontar los árboles para ensanchar el trazado vial, es necesario evaluar el impacto general. Este ejercicio implica no solo reconocer el árbol y lo que lo rodea sino situarlo en una complejidad socio-ecológicas humana y no humana. Este movimiento del pensamiento continúa a lo largo de toda la elaboración del EsIA.

Compartimos otro ejemplo. En 2016, frente a la consigna de describir el entorno del proyecto, un grupo presentó un mapa catastral y un texto con una descripción del lote donde se localizaría el proyecto.

A la pregunta respecto a qué elementos se encontraban en ese entorno, la respuesta fue «Nada, es una zona baldía» (Extracto de expresión estudiantil). Seguidamente, reforzamos la pregunta con imágenes satelitales que mostraban una zona arbolada, y nuevamente la respuesta fue «Nada, está baldía». Ante nuestra sorpresa, preguntamos explícitamente si consideraban que toda el área que se veía arbolada era «Nada» y por qué consideraban eso como 
algo «baldío». En esta ocasión, las/os estudiantes se detuvieron a pensar para finalmente señalar que consideraban que estaba baldío porque no tenía ocupación productiva.

Les solicitamos entonces a las/os estudiantes la realización de una observación presencial en la zona para reconocer el entorno, ya que esta acción es un requisito curricular para el Proyecto Final. Tras la visita, el grupo describió que el lugar contaba con gran cantidad de especies de árboles añosos, había un potrero con caballos y personas que los cuidaban. Dado que estas personas no poseían el título de propiedad, no consideran de relevancia dicha actividad productiva.

A partir de esta descripción, reflexionamos conjuntamente respecto a las características singulares que tenía este lote: actividades productivas de esas personas y la situación dominial. Ambas cuestiones han de ser consideradas para la planificación de un proyecto de obra de ingeniería dado que requiere contemplar las instancias jurídico-administrativas y el impacto en la supervivencia de una población local. Finalmente, estas situaciones pueden generar no solo mayores impactos socioeconómicos, legales y por ende ambientales, sino también conflictos socioambientales. En términos didácticos, era una oportunidad para comprender la situación compleja de esa área, tanto ecosistémica como social, económica y jurídicamente. A la vez, la necesidad de tener presentes las leyes y regulaciones vinculadas a la ejecución de una obra.

Esta experiencia fue muy enriquecedora para el grupo y el cuerpo docente, dado que nos permitió, primero, comprender de modo práctico la mirada de la complejidad socioambiental, y por otro lado, abrió la pregunta respecto a la necesidad de realizar el proyecto en ese espacio dado el impacto socioambiental que ya suponíamos era muy alto. La resolución fue relocalizar el proyecto a un área más favorable en términos ambientales, sociales y jurídicos.

En este ejemplo, podemos visualizar el segundo momento de desestabilización de saberes dado que aparece una pregunta que suele no realizarse a lo largo de la carrera de Ingeniería Civil, que tiene que ver con la necesidad social de un proyecto de obra de ingeniería. Este interrogante desnaturaliza la noción de que las obras de ingeniería de por sí son necesarias porque permiten el desarrollo económico de una región o territorio. En este sentido, se cuestiona si ese tipo de desarrollo es necesario, para qué y para quiénes. Debate fundamental para avanzar en la conceptualización y puesta en práctica del desarrollo sustentable.

Por otra parte, en este momento los/las estudiantes visualizan las consecuencias, efectos o impactos socioambientales de las obras antes de que estas se realicen y no a posteriori, cuando ya hay impactos irreversibles, de 
manera que, dado que el proyecto no ha sido realizado, pueden encontrarse las alternativas que eviten daños, impactos altos o irreparables.

\section{Conclusiones}

En este artículo hemos sistematizado la elaboración de la estrategia didáctica-pedagógica de la «Simulación de Estudio de Impacto Ambiental» en el contexto de la formación académica para la ingeniería civil en una universidad pública de Argentina, la UTN.

Esta estrategia nos resulta adecuada para la puesta en práctica de los dos objetivos de la EA ya explicitados. Por un lado, es un instrumento educativo que prepara a los/as estudiantes para comprender situaciones complejas; en otras palabras, comprender teórica y prácticamente el pensamiento de la complejidad. En segundo lugar, como mencionamos, esta Simulación se enmarca en la realización del Proyecto Final que las/os estudiantes realizan en conjunto con actores gubernamentales y sociales y con diferentes docentes universitarios/as. De modo que esta situación es un suelo fértil para promover la posición ética profesional hacia el desarrollo sustentable.

La elaboración de la Simulación del EsIA resulta una estrategia didáctica adecuada a partir de la cual confeccionar una visión integral del ambiente, considerándolo en sus múltiples y complejas relaciones, comprendiendo aspectos físicos, culturales, bióticos, tecnológicos, históricos, políticos, económicos, éticos, etc., a la vez que entender a la realidad como construcción social en la que confluyen dimensiones políticas, sociales, culturales, ecológicas, atravesadas por relaciones de poder a lo largo del tiempo y puestas en práctica por diferentes sujetos sociales.

Somos conscientes de que este ejercicio se propone en términos ideales, dado que las/os estudiantes tienen libertad, e incluso lo fomentamos, de que piensen y elaboren alternativas sustentables en sus proyectos, que puedan evaluar los impactos y mitigaciones e incluso proponer cambios desde el inicio de la propuesta. La Simulación es ideal dado que hacemos un «como si» en la realidad todo eso pudiera suceder de manera armónica con un consenso gubernamental-privado-social de buscar alternativas para la sustentabilidad. Nos parece adecuado plantear un escenario ideal dentro de lo posible ya que es una instancia de formación académica y también porque la experiencia de pensar lo imposible abre nuevas alternativas.

La actividad de elaboración de la Simulación del EsIA del Proyecto Final no solo persigue el fin específico de saber si el balance de impacto ambiental de un proyecto de ingeniería civil es positivo o negativo, ni siquiera de apro- 
piarse del método que se propone para su análisis, sino que principalmente se procura que las/os estudiantes consideren su ejercicio profesional como una práctica social que demanda una mirada crítica sobre las decisiones en torno a la ejecución de un proyecto.

Durante estos años de práctica docente, se ha observado que distintos grupos de estudiantes han logrado comprender las diversas implicancias socio-ecológicas inherentes a su Proyecto Final.

Al respecto, constatamos que en la formación en ingeniería civil tradicional aún persiste una mirada tecnicista, tanto en docentes y estudiantes, asociada a las asignaturas de fuerte contenido técnico-científico. Este enfoque implica desconocer el contexto sociocultural en el que se inserta la formación profesional, así como también sobrevalorar el factor económico que enfatiza la perspectiva productivista tradicional. Interrogar esta mirada tecnicista se traduce en el primer momento de ruptura de las/os estudiantes y de encuentro con la incertidumbre que implica pensar desde el paradigma de la complejidad.

En tanto concebimos el aprendizaje como una construcción social, la estrategia didáctica-pedagógica abordada promueve la participación en prácticas sociales histórica y culturalmente situadas; por lo tanto, consideramos que la formación como sujetos activos de una comunidad permite configurarse como miembro y participar de su producción (Lave y Packer, 2011, en Malagón Patiño, 2018).

En este sentido, a lo largo de estos años de poner en práctica esta estrategia, hemos reflexionado que la interacción entre estudiantes y cuerpo docente dentro del espacio áulico potencia, por un lado, la producción colectiva de saberes y subjetividades. Por otro, la intervención equitativa en diferentes órdenes de la sociedad, desde lo ambiental, lo político, lo económico, lo científico, y la problematización de las desigualdades sociales, ambientales, económicas, de clase, raza o género.

Finalmente, cuestionar la mirada tecnicista preponderante en la formación académica para la ingeniería civil implica promover una perspectiva ético-política hacia la sustentabilidad en el ejercicio profesional. El paradigma tradicional de la ingeniería civil asociado a la mirada técnica promueve la construcción del proyecto de obra y luego la evaluación de su impacto socio-ecológico. En nuestra experiencia, la elaboración de la Simulación del EsIA permite que las/os estudiantes interroguen sus Proyectos Finales sobre el impacto socio-ecológico durante la realización y proyección de los mismos.

Así planteada, la búsqueda de modificar el proyecto en función de una perspectiva de Educación Ambiental para el Desarrollo Sustentable debería 
ir transformándose en pensar el proyecto considerando esa mirada desde su concepción como idea.

Este recorrido permite situar el rol de las/os ingenieras/os civiles en la construcción de la realidad social desde una mirada de la complejidad ambiental, y hace posible una reflexión y práctica de una ética que promueva un futuro sustentable.

\section{Notas}

1. Congreso Internacional INGENIERÍA 2010

- ARGENTINA: «Tecnología, Innovación y Producción para el Desarrollo Sostenible». Disponible en: https://www.ingenieria2010. com.ar/ [29/5/2020]. « volver

\section{Referencias bibliográficas}

CARRASCO, J. (2004). Una didáctica para hoy. Cómo enseñar mejor. Madrid: Rialp.

DI PACE, M.; Crojethovich, M. A., y Ruggerio, C. (2012). Paradigmas Ambientales. En M. D. Bartrons, Ecología Urbana, 335-367. Los Polvorines: Editorial Universidad Nacional General Sarmiento.

FERNÁNDEZ-VÍTORA, V. C. (1993). Guía metodológica para la Evaluación de Impacto Ambiental. Madrid: Ediciones Mundi-Prensa.

FERRANTI, L. y Orellana, J. (2019). El estudio de casos en Ingeniería Civil. Una estrategia didáctica para la formación ambiental. Editorial Académica Española.

FERRER, D.; et al. (2008). An international comparative analysis of sustainability transformation across seven universities. International Journal of Sustainability in Higher Education, 295-316.

GALLOPIN, G. (2003). Sostenibilidad y Desarrollo Sostenible: un enfoque sistémico.
2. Cabe aclarar que el actual equipo de cátedra se forma en el año 2016, siguiendo la tradición del ingeniero civil Osvaldo Russo, su mentor, y la arquitecta María Graham, profesora adjunta hasta ese año. « volver

Serie Medio Ambiente y Desarrollo, publicación de las Naciones Unidas - CEPAL, n. 64 .

GÓMEZ OREA, D. (2002). Evaluación de Impacto Ambiental. Madrid: Ediciones Mundi-Prensa.

GUDYNAS, E. (2004). Cap. 3 «Una mirada histórica al desarrollo sostenible». En E. Gudynas, Ecología, Economía y Ética del Desarrollo Sostenible, 47-66. Montevideo: Coscoroba del Centro Latino Americano de Ecología Social (CLAES).

HAESBAERT, R. (2011). El mito de la desterritorialización. México: Siglo XXI.

HARVEY, D. (2000). Espacios de esperanza. Madrid: Akal ediciones.

LEFEBVRE, H. (1974). La producción del espacio. Revista de Sociología, n. ${ }^{\circ} 3,[\mathrm{~s} / \mathrm{d}]$.

LEFF, E. (1998a). Educación ambiental y desarrollo sustentable. En Formación Ambiental, 9-10. México: PNUMA. 
LEFF, E. (1998b). Saber ambiental, sustentabilidad, racionalidad, complejidad, poder. México: Siglo XXI Editores.

LEFF, E. (2014). La apuesta por la vida. Buenos Aires: Siglo XXI.

MALAGÓN PATIÑO, M. R. (2018). Concepciones sobre el aprendizaje y su relación con las prácticas pedagógicas. Informador Técnico, 108-119.

MORIN, E. (1990). Introducción al pensamiento complejo. Barcelona: Gedisa.

PORTO GONÇALVEZ, C. W. (2004). El desafío ambiental. México: Programa de las Naciones Unidas para el Medio Ambiente.

SWYNGEDOUW, E. (02 de 09 de 2009). La economía política y la ecología política del ciclo hidrosocial. Obtenido de https://doi. org/10.1111/j.1936-704X.2009.00054.x

TORRES PERNALETE, M. R. (2012). El compromiso social de las universidades: Alternativas para afianzar una educación para el desarrollo sustentable. En N. Asili Pierucci, Vida sustentable. La experiencia de un sueño compartido, 141-182. Puebla, México: Fundación de la Universidad de las Américas.

WASSERMAN, S. (1999). El estudio de casos como método de enseñanza. Buenos Aires: Amorrortu.

\section{Documentos de organismos gubernamentales} SECRETARÍA DE AMBIENTE Y DESARROLLO SUSTENTABLE (2018). Guía para la elaboración de un Estudio de Impacto Ambiental. Disponible en: https://www.argentina.gob.ar/ ambiente/sustentabilidad/evaluacion-ambiental/impacto/guia-elaboracion-esia

SECRETARÍA DE AMBIENTE Y DESARROLLO SUSTENTABLE (2009). «Educación ambiental: Aportes políticos y pedagógicos en la construcción del campo de la Educación Ambiental», Buenos Aires: Secretaría de Ambiente y Desarrollo Sustentable. Disponible en: https://web.ua.es/es/giecryal/documentos/educacion-ambiental.pdf 